\title{
Demonstrating a Continuous Set of Two-Qubit Gates for Near-Term Quantum Algorithms
}

\author{
B. Foxen $\odot,{ }^{1,2}$ C. Neill, ${ }^{2}$ A. Dunsworth, ${ }^{2}$ P. Roushan, ${ }^{2}$ B. Chiaro, ${ }^{1}$ A. Megrant ${ }^{2}$ J. Kelly, ${ }^{2}$ Zijun Chen, ${ }^{2}$ \\ K. Satzinger, ${ }^{2}$ R. Barends, ${ }^{2}$ F. Arute, ${ }^{2}$ K. Arya, ${ }^{2}$ R. Babbush, ${ }^{2}$ D. Bacon, ${ }^{2}$ J. C. Bardin, ${ }^{2,3}$ S. Boixo, ${ }^{2}$ D. Buell, ${ }^{2}$ \\ B. Burkett, ${ }^{2}$ Yu Chen, ${ }^{2}$ R. Collins, ${ }^{2}$ E. Farhi, ${ }^{2}$ A. Fowler, ${ }^{2}$ C. Gidney, ${ }^{2}$ M. Giustina, ${ }^{2}$ R. Graff, ${ }^{2}$ M. Harrigan, ${ }^{2}$ \\ T. Huang, ${ }^{2}$ S. V. Isakov, ${ }^{2}$ E. Jeffrey, ${ }^{2}$ Z. Jiang, ${ }^{2}$ D. Kafri, ${ }^{2}$ K. Kechedzhi, ${ }^{2}$ P. Klimov, ${ }^{2}$ A. Korotkov, ${ }^{2}$ \\ F. Kostritsa, ${ }^{2}$ D. Landhuis, ${ }^{2}$ E. Lucero, ${ }^{2}$ J. McClean, ${ }^{2}$ M. McEwen, ${ }^{1}$ X. Mi, ${ }^{2}$ M. Mohseni, ${ }^{2}$ J. Y. Mutus, ${ }^{2}$ \\ O. Naaman, ${ }^{2}$ M. Neeley, ${ }^{2}$ M. Niu, ${ }^{2}$ A. Petukhov, ${ }^{2}$ C. Quintana, ${ }^{2}$ N. Rubin, ${ }^{2}$ D. Sank, ${ }^{2}$ V. Smelyanskiy, ${ }^{2}$ \\ A. Vainsencher, ${ }^{2}$ T. C. White, ${ }^{2}$ Z. Yao, ${ }^{2}$ P. Yeh, ${ }^{2}$ A. Zalcman, ${ }^{2}$ H. Neven, ${ }^{2, *}$ and J. M. Martinis ${ }^{1,2}$ \\ (Google AI Quantum) \\ ${ }^{1}$ Department of Physics, University of California, Santa Barbara, California 93106, USA \\ ${ }^{2}$ Google Research, Santa Barbara, California 93117, USA \\ ${ }^{3}$ Department of Electrical and Computer Engineering, University of Massachusetts-Amherst, Amherst, Massachusetts 01003, USA
}

(Received 29 January 2020; revised 27 June 2020; accepted 22 July 2020; published 15 September 2020)

\begin{abstract}
Quantum algorithms offer a dramatic speedup for computational problems in material science and chemistry. However, any near-term realizations of these algorithms will need to be optimized to fit within the finite resources offered by existing noisy hardware. Here, taking advantage of the adjustable coupling of gmon qubits, we demonstrate a continuous two-qubit gate set that can provide a threefold reduction in circuit depth as compared to a standard decomposition. We implement two gate families: an imaginary swap-like (iSWAP-like) gate to attain an arbitrary swap angle, $\theta$, and a controlled-phase gate that generates an arbitrary conditional phase, $\phi$. Using one of each of these gates, we can perform an arbitrary two-qubit gate within the excitation-preserving subspace allowing for a complete implementation of the so-called Fermionic simulation (fSim) gate set. We benchmark the fidelity of the iSWAP-like and controlled-phase gate families as well as 525 other fSim gates spread evenly across the entire $\operatorname{fSim}(\theta, \phi)$ parameter space, achieving a purity-limited average two-qubit Pauli error of $3.8 \times 10^{-3}$ per fSim gate.
\end{abstract}

DOI: 10.1103/PhysRevLett.125.120504

Introduction.-Quantum computing is a potentially transformative technology, but challenges remain in identifying a path toward solving practical problems with a quantum advantage [1]. Continued progress toward this goal may be made on many fronts, including qubit coherence or scalability [2,3], measurement or gate fidelities [4,5], and algorithmic improvements that reduce the required circuit depth through compilation [6]. In superconducting qubits, single-qubit gates usually exhibit a factor of 2 or more lower error than two-qubit gates. Consequently, a typical strategy has been to demonstrate a minimally universal gate set consisting of arbitrary singlequbit rotations and a single two-qubit gate [7]. This is an efficient approach for some algorithms, including surface code error correction, which compile optimally with such a

Published by the American Physical Society under the terms of the Creative Commons Attribution 4.0 International license. Further distribution of this work must maintain attribution to the author(s) and the published article's title, journal citation, and DOI. gate set [8]. However, many noisy intermediate-scale quantum (NISQ) [9] algorithms require a more diverse set of two-qubit gates. An implementation of these gates could take the place of six to eight single-qubit gates and three $\mathrm{CZ}_{\phi}$ gates per arbitrary two-qubit gate required with an optimal decomposition into a minimally universal gate set [10].

In the NISQ era, we need the largest two-qubit gate set that may be implemented with high fidelity. A general twoqubit unitary gate allows independent control over the strength of $\sigma_{X} \sigma_{X}, \sigma_{Y} \sigma_{Y}$, and $\sigma_{Z} \sigma_{Z}$ coupling between qubits requiring both DC and microwave control of gmon qubits [11]. However, models of interacting particles typically conserve the number of excitations corresponding to a simpler model where the $\sigma_{X} \sigma_{X}$ and $\sigma_{Y} \sigma_{Y}$ couplings have equal coefficients. This reduces the number of control parameters from three to two and eliminates the need for microwave control during an algorithm. This set of excitation-conserving gates has been appropriately termed the Fermionic simulation (fSim) gate set since it maps electron conservation in a chemistry problem to photon conservation in qubits [12]. An fSim gate can be defined 
with two control angles: $\theta$, the $|01\rangle \leftrightarrow|10\rangle$ swap angle, and, $\phi$, the phase of the $|11\rangle$ state with a matrix representation in the $|00\rangle,|01\rangle,|10\rangle,|11\rangle$ basis given by

$$
\mathrm{fSim}(\theta, \phi)=\left(\begin{array}{cccc}
1 & 0 & 0 & 0 \\
0 & \cos \theta & -i \sin \theta & 0 \\
0 & -i \sin \theta & \cos \theta & 0 \\
0 & 0 & 0 & e^{-i \phi}
\end{array}\right)
$$

We use this as both a convenient definition and a useful model for describing general two-qubit gates resulting from arbitrary flux control of gmon qubits. Notably, promising low-depth algorithms using this gate set have been proposed, including the quantum approximate optimization algorithm [13] and an algorithm for linear-depth circuits simulating the electronic structure of molecules [12]. Additionally, algorithms performed with just $\mathrm{z}$ rotations and fSim gates enable error mitigation techniques, including postselection and zero noise extrapolation [14], further improving this gate set's prospects on NISQ processors.

Here, we first demonstrate the strong flux tunable coupling between gmon qubits that we use to perform fast two-qubit gates. Then, to describe our calibration and control strategy, we use shallow circuits to illuminate the natural correspondence of the coupled transmon Hamiltonian and the fSim gate set. We use cross-entropy benchmarking (XEB) [15] to characterize two linearly independent and continuous families of entangling gates: the imaginary swap-like (iSWAP-like) family corresponding to $\operatorname{fSim}\left(\theta, \phi \propto \theta^{2}\right)$, and the controlled-phase (CPHASE) family corresponding to $\operatorname{fSim}\left(\theta \approx 0^{\circ}, \phi\right)$. We then combine these two continuous gate sets to calibrate and benchmark 525 fSim gates spread evenly across the entire $(\theta, \phi)$ parameter space.

Strong coupling with gmon qubits.-The quantum processors used in this work followed the Sycamore design used in reference [16], with each processor consisting of a chain of four gmon transmon qubits coupled by three transmon qubit couplers. Both the qubit frequencies and their coupling can be independently controlled, providing several advantages over fixed coupling designs $[11,17,18]$. First, since we can turn off the coupling at any detuning, both qubits may idle and perform single-qubit gates while operating closer to their flux insensitive point. This improves dephasing and decreases our sensitivity to flux settling tails. Second, since entangling gates are performed by bringing the qubit states near resonance, idling the qubits closer together means that gates require much smaller dynamic detunings, further reducing the amplitude of flux settling tails $[19,20]$. Third, since the minimum coupling strength is not dependent on the maximum qubitqubit detuning, we are able to increase the overall maximum coupling strength, enabling faster gates with reduced decoherence error.

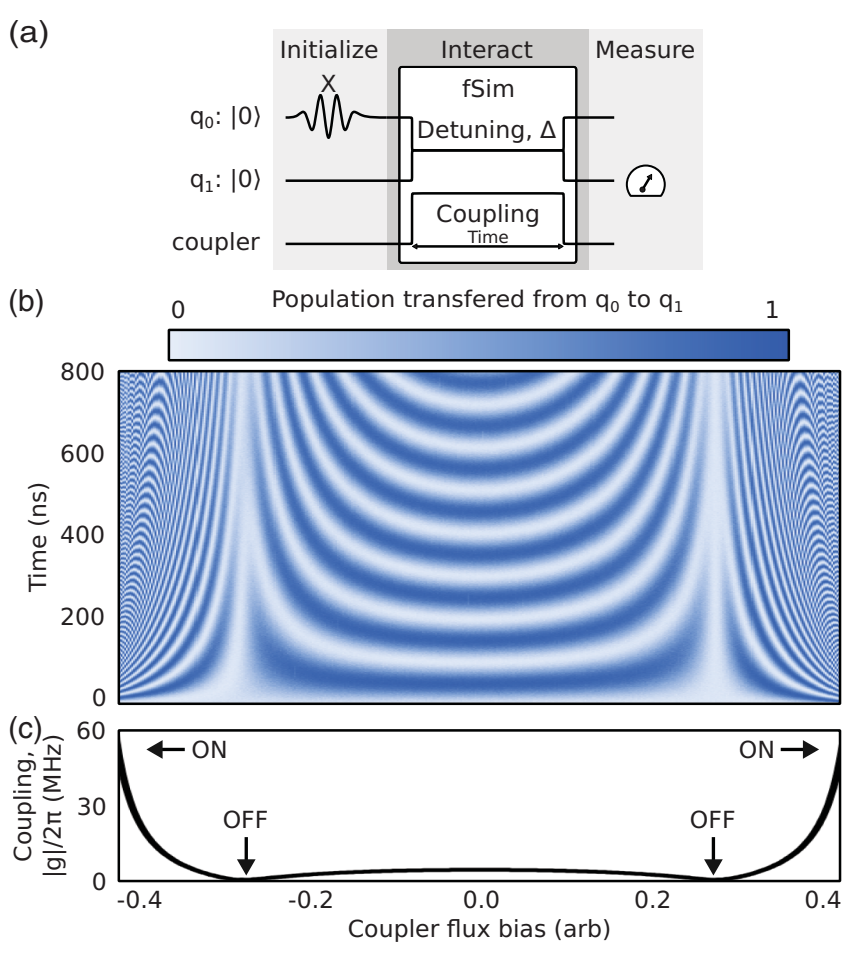

FIG. 1. Demonstration of the tunable coupling between gmon qubits. (a) Pulse sequence used to measure swapping as a function of coupler bias. We initialize one qubit, perform an fSim gate defined by a set of three flux pulses that control the qubit frequencies and the coupling between the qubits, and measure the population of the other qubit. (b) We vary the fSim gate as a function of the length and amplitude of the coupler pulse to measure the swap rate as a function of the bias amplitude. (c) By taking the Fourier transform of the oscillations in (b), we extract the coupling strength, $|g|$, as a function of coupler bias. The coupling changes sign at the two "OFF" biases, ensuring we can turn the coupling off.

In Fig. 1, we characterize the qubit-qubit coupling strength as a function of the coupler flux bias. Using the pulse sequence in Fig. 1(a), we initialize one qubit, apply an fSim gate, and measure the population transferred to the other qubit. Each fSim gate is defined by the amplitude and duration of three, nominally rectangular, flux bias pulses. Two pulses control the qubit frequencies and set their relative detuning, $\Delta$, while the third pulse controls the coupling strength between the qubits, $g$. In Fig. 1(b), we repeat this pulse sequence using the qubit flux biases to place them on resonance $(\Delta=0 \mathrm{MHz})$ while varying the coupler bias amplitude and the shared duration of all three pulses. By taking the Fourier transform of the oscillating population data in Fig. 1(b), we extract the swap rate as a function of coupler bias, which is equivalent to twice the qubit-qubit coupling, $g$, plotted in Fig. 1(c). We measure $g / 2 \pi=6 \mathrm{MHz}$ when the coupler is at its maximum frequency (zero bias) and a coupling exceeding $-50 \mathrm{MHz}$ as the coupler frequency is reduced. The net coupling changes sign between these two regions, ensuring we can 
turn the coupling off. During general operation, we idle and perform single-qubit gates with the coupler at the "OFF" bias and make excursions to stronger couplings ("ON" region) to perform fSim gates. In this work, we use $g_{\max } / 2 \pi \approx-45 \mathrm{MHz}$, which is three times stronger than is typical for fixed coupling devices.

Coupled transmon physics and the fSim gate set.-In the absence of a resonant microwave drive, coupled transmon qubits naturally evolve within the excitation-preserving subspace. The specific time evolution is determined by three parameters: the qubit nonlinearity, $\eta$; the qubit-qubit frequency detuning, $\Delta$; and the coupling between qubits, $g$. While $\eta$ is fixed at $240 \mathrm{MHz}$ by qubit capacitance, the gmon architecture allows for time-dependent control of both $\Delta$ and $|g|$ using DC to $\sim 200 \mathrm{MHz}$ bandwidth flux waveforms. The average of the two qubit frequencies during the interaction is a free parameter that may be used to avoid coupled two level system defects present in the frequency spectrum of either qubit [21-23]. For simplicity, we limit our fSim control pulses to synchronous, nominally rectangular waveforms (see Supplemental Material [24], Sec. IV) defined by four parameters: a shared length, typically 13 to $15 \mathrm{~ns}$, and three control amplitudes that set $g$ and $\Delta$. While further pulse shaping may improve gate performance in the future, these basic waveforms were sufficient to approach the decoherence limit of our qubits, which have a $\mathrm{T}_{1}$ of $25.3 \pm 7.3 \mu$ s (Supplemental Material [24], Sec. III B).

The full fSim control model describes any low-leakage two-qubit unitary evolution with five parameters: $\theta$ and $\phi$, discussed previously, in addition to three parameters describing single-qubit phases as detailed in Sec. I of the Supplemental Material [24]. Here, we focus on the parameters that describe the two-qubit interaction and use the three experiments, described in Fig. 2, to measure leakage to the $|02\rangle$ state and map out the $\phi$ and $\theta$ control landscape (the complete unitary tomography procedure is outlined in Sec. III C of the Supplemental Material [24]). Each experiment follows the same pattern: initialize a relevant state, apply fSim control pulses, and then perform either population or tomographic measurements to extract the desired qubit's population or phase. Within the fSim model, leakage is the dominant error. In Fig. 2(a), we map out leakage by initializing $|11\rangle$ and measuring the $|0\rangle$ population of the lower frequency qubit as a proxy for leakage in the higher frequency qubit. In Fig. 2(b), we explore the $\phi$ parameter space by performing a Ramsey experiment where we take the difference in the (a)

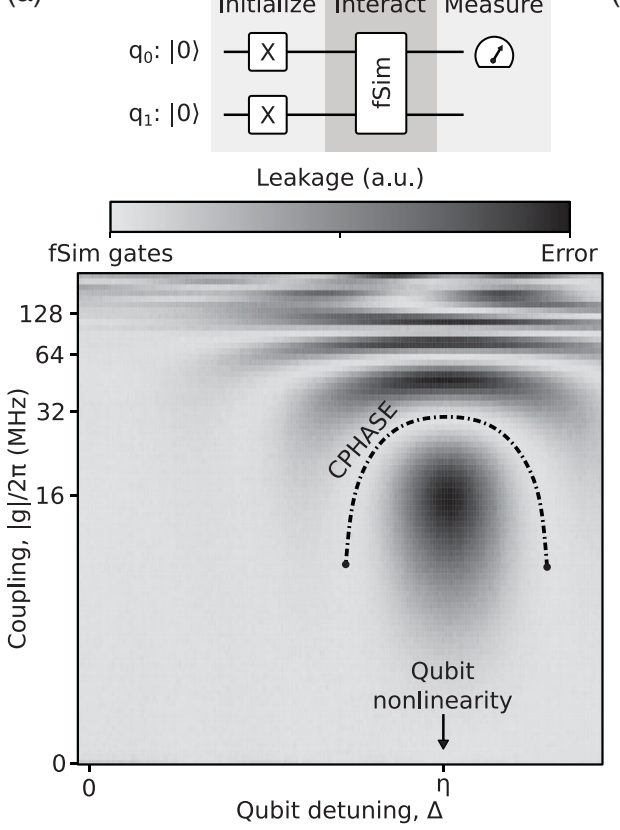

(b)

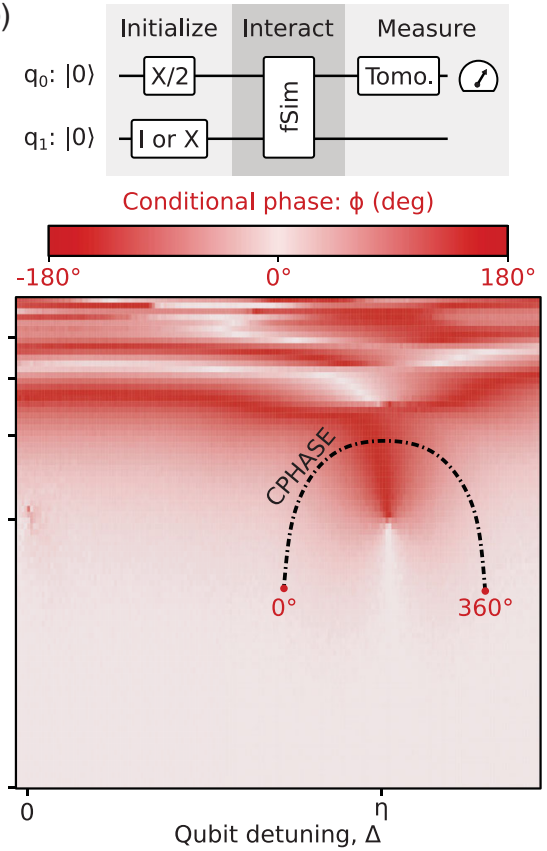

(c)
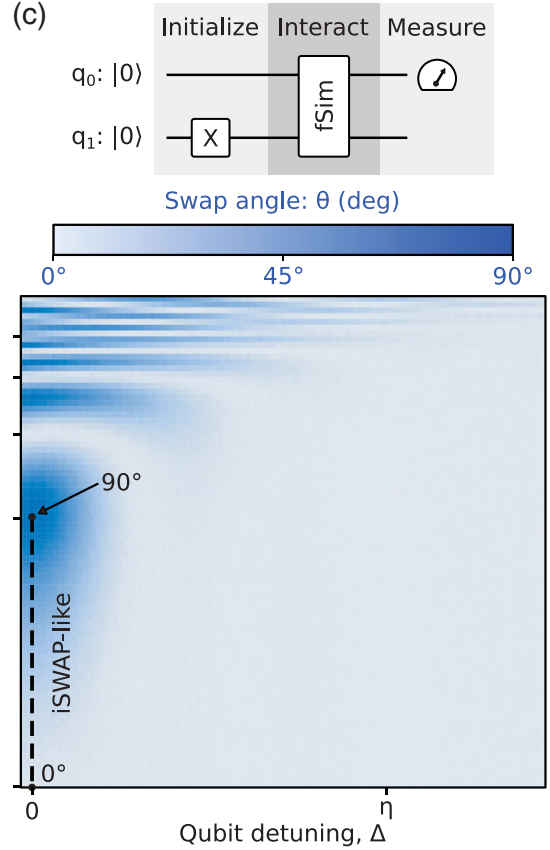

FIG. 2. Exploring the parameter space of two-qubit gates. Each pixel represents one experiment. We use a set of 15 ns rectangular current bias waveforms to perform some fSim unitary by setting the qubit-qubit detuning, $\Delta$, and the coupling strength, $g$. (a) To identify the low-leakage gates described by the fSim model, we measure leakage by initializing the $|11\rangle$ state and measuring the $|02\rangle$ state. When the detuning is near the qubit nonlinearity, we observe the expected Rabi oscillations. (b) We measure the conditional phase, $\phi$, by performing a Ramsey experiment where we initialize one qubit with an $\mathrm{X} / 2$ gate and perform tomography to measure the difference in accumulated phase $(\phi)$ with and without initializing the other qubit to the $|1\rangle$ state. By choosing combinations of $\Delta$ and $g$ as indicated by the CPHASE dash-dotted line [chosen as the low-leakage coupling strength from (a)], we are able to achieve any $\phi:\left[-180^{\circ}, 180^{\circ}\right]$. (c) We measure the swap angle, $\theta$, by initializing the $|01\rangle$ state and measuring the $|10\rangle$ state. By placing the qubits on resonance and varying the coupling strength along the iSWAP-like dashed line, we are able to achieve any $\theta:\left[0^{\circ}, 90^{\circ}\right]$. 
accumulated phase with and without the second qubit initialized to the $|1\rangle$ state. Finally, in Fig. 2(c), we explore the $\theta$ parameter space by initializing one qubit to the $|1\rangle$ state and measuring the $|1\rangle$ population of the other qubit after the fSim gate. The Rabi oscillation physics explored with these measurements is reproduced with fairly rudimentary numerics in Sec. II of the Supplemental Material [24], but these experiments serve to demonstrate our fSim control strategy.

Benchmarking iSWAP-like and CPHASE gates.-The data presented in Fig. 2 provide a map for implementing an arbitrary fSim - each pixel defines a set of three control amplitudes, and any control amplitudes yielding low leakage should result in a high-fidelity gate described by the fSim control model [Eq. (1)]. While it may be possible to perform an arbitrary fSim gate with a single set of flux pulses using either very strong coupling or more complex control waveforms, we have chosen to implement an arbitrary fSim gate as a composition of two continuous gate families using simple rectangular control pulses to minimize the gate length. The first gate family completes a diabatic $|11\rangle \rightleftarrows|02\rangle$ swap to perform a gate with an arbitrary conditional phase, $\phi$, using control amplitudes denoted by the dot-dashed line labeled "CPHASE" in Figs. 2(a) and 2(b). The dominant control angle in the CPHASE gate family is the conditional phase, but we do accumulate a small swap angle $\theta$ due to the strong coupling necessary to perform a fast CPHASE gate $\left(\theta \leq 5^{\circ}\right.$ for a 13 ns CPHASE gate - this may be reduced by increasing the gate duration). The second gate family places the qubits on resonance $(\Delta=0 \mathrm{MHz})$ and varies $g$ to reach the desired swap angle, $\theta$, using control amplitudes along the dashed line labeled "iSWAP-like" in Fig. 2(c). We have deemed this gate family "iSWAP-like" since the swap angle varies from $\theta:\left[0^{\circ}, 90^{\circ}\right]$ and because this gate accumulates a conditional phase $\phi \propto \theta^{2}$ due to the dispersive interaction with the $|02\rangle$ and $|20\rangle$ states. Both of these gates are a subset of the fSim group individually, and, compiled together, they can reach the full fSim parameter space.

In Fig. 3, we characterize both the iSWAP-like and CPHASE gate families using XEB [15]. On the left axes, we plot the optimized values of $\theta$ and $\phi$ for a range of CPHASE and iSWAP-like gates, and on the right y axes, we plot the Pauli error per two-qubit gate (Supplemental Material [24], Sec. III B), achieving average errors of $1.9 \times 10^{-3}$ and $1.2 \times 10^{-3}$ for each gate family, respectively.

Benchmarking fSim gates.-In Fig. 4(a), we present the Pauli error of 525 distinct $\mathrm{fSim}(\theta, \phi)$ gates where the values of $\theta$ and $\phi$ have been constrained to be exactly the values indicated by the xy coordinates at the center of each pixel (where ex situ optimization has been used to optimize only the single-qubit phases). Each $28 \mathrm{~ns}$ long fSim gate in Fig. 4 is a composition of a 15 ns CPHASE gate followed by a $13 \mathrm{~ns}$ iSWAP-like gate. While the fSim fidelity is
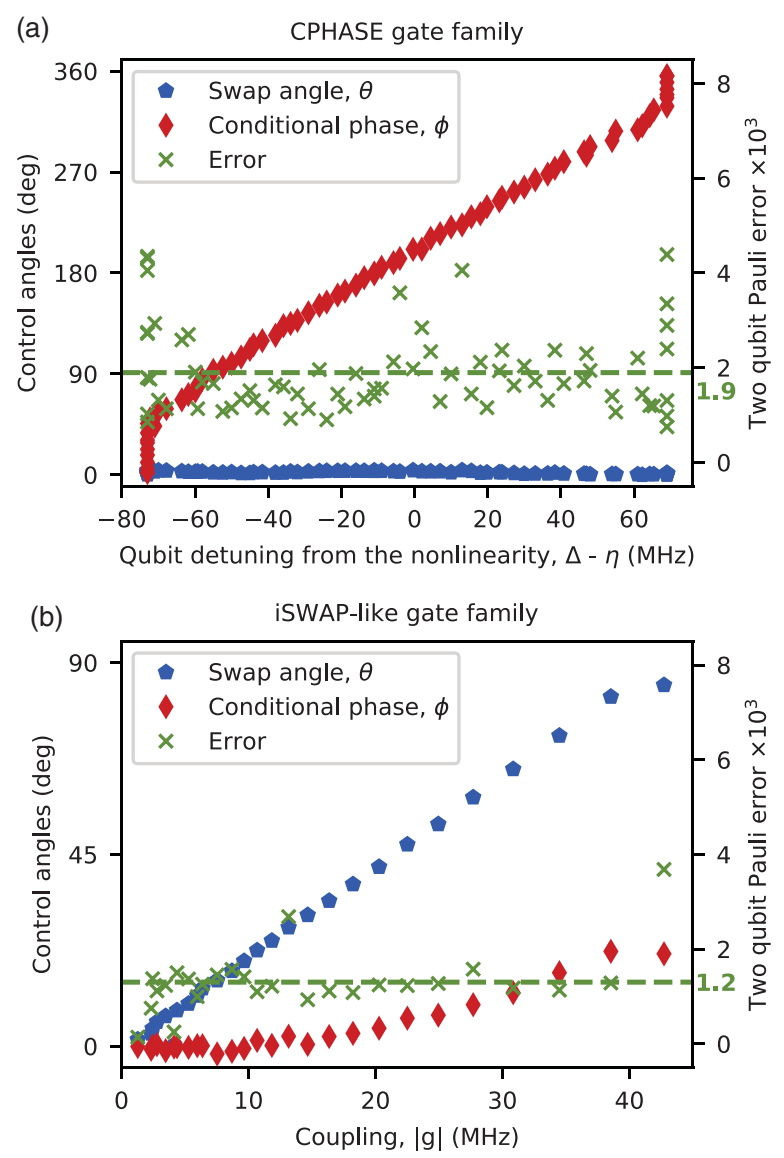

FIG. 3. Characterizing the iSWAP-like and CPHASE gate families with cross-entropy benchmarking. We plot the optimized fSim control angles, $\theta$ and $\phi$, on the left y-axes and the Pauli gate error per two-qubit gate on the right y-axes, conservatively assuming $7.5 \times 10^{-4}$ single-qubit Pauli gate errors. (a) Characterization of the CPHASE gate family corresponding to $\mathrm{fSim}\left(\theta \approx 0^{\circ}, \phi\right)$. Each gate is $15 \mathrm{~ns}$ long, consisting of control pulses that vary the qubit detuning, $\Delta$, around the qubit nonlinearity, $\eta$, with a coupler bias amplitude chosen to complete one full swap: $|11\rangle \rightarrow|02\rangle \rightarrow|11\rangle$. We measure an average two-qubit Pauli error of $1.9 \times 10^{-3}$ for the CPHASE family. (b) Characterization of the iSWAP-like gate family corresponding to $\operatorname{fim}\left(\theta, \phi \propto \theta^{2}\right)$. Each gate is $13 \mathrm{~ns}$ long, consisting of control pulses that place the qubits on resonance and vary the coupling strength, $|g|$, to achieve an arbitrary swap angle $\theta$ between the $|01\rangle$ and $|10\rangle$ states. We measure an average two-qubit Pauli error of $1.2 \times 10^{-3}$ for the iSWAP-like family.

largely independent of the values of $\theta$ and $\phi$, there are a few features of note. As discussed in Sec. IVC of the Supplemental Material [24], we most directly calibrated line cuts at $\theta=0^{\circ}, 90^{\circ}$ and $\phi=180^{\circ}$. The regions of higher error where $\phi$ is near $0^{\circ}\left(360^{\circ}\right)$ involve the most extrapolation from the directly calibrated control amplitudes. There is also a faintly visible indication of a band of higher error near $\phi \approx 240^{\circ}$, which we believe is due to a weakly interacting two level system defect in the spectrum of one of the qubits. In the future, we hope to avoid such defects 


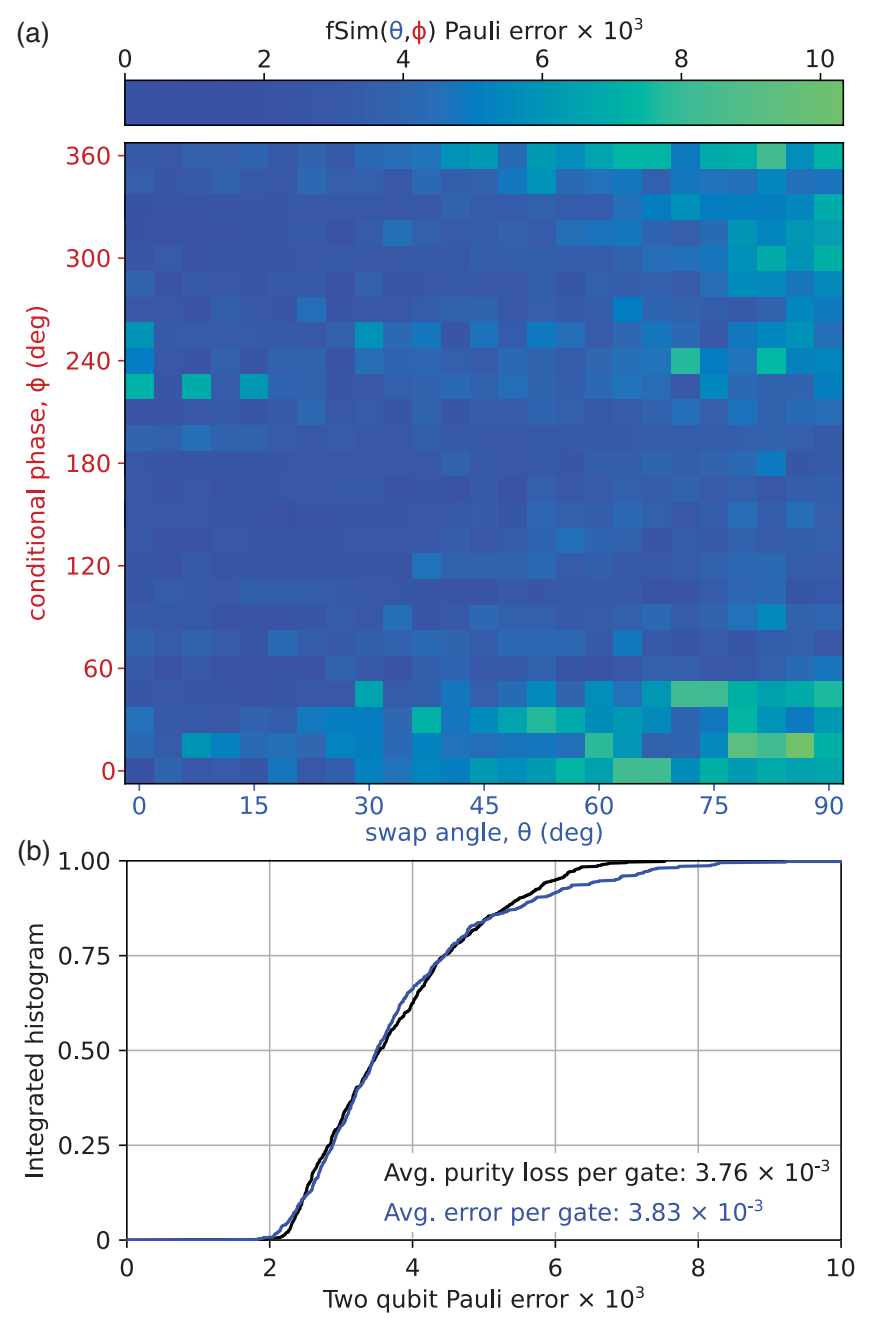

FIG. 4. Benchmarking the fSim gate set. Using XEB, we measure the Pauli error per cycle and subtract off a conservative estimate for the single-qubit Pauli gate errors of $7.5 \times 10^{-4}$. (a) We plot the two-qubit Pauli error for 525 fSim gates where $\theta$ and $\phi$ have been constrained to a grid. (b) Histogram of both the error and purity loss for each gate presented in (a). Here we confirm a purity (coherence) limited average error for our fSim gates of $3.83 \times 10^{-3}$.

by shifting the frequencies of both qubits while maintaining their relative detuning. In Fig. 4(b), we histogram these results in addition to the purity [31] loss per fSim and confirm a purity-limited average Pauli error of $3.83 \times 10^{-3}$ per fSim gate.

Conclusions.-We have implemented continuous iSWAP-like and CPHASE gate families with average Pauli error rates of $1.2 \times 10^{-3}$ and $1.9 \times 10^{-3}$, respectively. These fast (13-15 ns) gates take advantage of the strong, tunable, qubit-qubit coupling offered by our gmon transmon qubit architecture, achieving error rates more than a factor of 2 lower than the best previously reported twoqubit gates for superconducting qubits [32]. Additionally, we have combined these two gate sets to demonstrate a complete implementation of the two-qubit fSim gate set with an average Pauli error of $3.83 \times 10^{-3}$ per gate. This direct implementation of the fSim gate offers roughly an additional factor of three in compilation efficiency for NISQ algorithms over a minimally universal gate set.

This work was supported by Google LLC. Gmon qubits were fabricated at the UC Santa Barbara Nanofabrication Facility, part of the NSF National Nanotechnology Infrastructure Network funded by NSF.

B. F., C. N., and A. D. contributed equally to this work.

*Corresponding author. neven@google.com

[1] R. P. Feynman, Simulating physics with computers, Int. J. Theor. Phys. 21, 467 (1982).

[2] J. Koch, T. M. Yu, J. Gambetta, A. A. Houck, D. I. Schuster, J. Majer, A. Blais, M. H. Devoret, S. M. Girvin, and R. J. Schoelkopf, Charge-insensitive qubit design derived from the cooper pair box, Phys. Rev. A 76, 042319 (2007).

[3] D. Rosenberg, D. Kim, R. Das, D. Yost, S. Gustavsson, D. Hover, P. Krantz, A. Melville, L. Racz, G. O. Samach, S. J. Weber, F. Yan, J. L. Yoder, A. J. Kerman, and W. D. Oliver, 3d integrated superconducting qubits, npj Quantum Inf. 3, 42 (2017).

[4] D. I. Schuster, A. Wallraff, A. Blais, L. Frunzio, R.-S. Huang, J. Majer, S. M. Girvin, and R. J. Schoelkopf, AC Stark Shift and Dephasing of a Superconducting Qubit Strongly Coupled to a Cavity Field, Phys. Rev. Lett. 94, 123602 (2005).

[5] E. Jeffrey, D. Sank, J. Y. Mutus, T.C. White, J. Kelly, R. Barends, Y. Chen, Z. Chen, B. Chiaro, A. Dunsworth, A. Megrant, P. J. J. O’Malley, C. Neill, P. Roushan, A. Vainsencher, J. Wenner, A. N. Cleland, and J. M. Martinis, Fast Accurate State Measurement with Superconducting Qubits, Phys. Rev. Lett. 112, 190504 (2014).

[6] F. T. Chong, D. Franklin, and M. Martonosi, Programming languages and compiler design for realistic quantum hardware, Nature (London) 549, 180 (2017).

[7] A. Barenco, C. H. Bennett, R. Cleve, D. P. DiVincenzo, N. Margolus, P. Shor, T. Sleator, J. A. Smolin, and H. Weinfurter, Elementary gates for quantum computation, Phys. Rev. A 52, 3457 (1995).

[8] A. G. Fowler, M. Mariantoni, J. M. Martinis, and A. N. Cleland, Surface codes: Towards practical large-scale quantum computation, Phys. Rev. A 86, 032324 (2012).

[9] J. Preskill, Quantum computing in the NISQ era and beyond, Quantum 2, 79 (2018).

[10] N. Khaneja and S. J. Glaser, Cartan decomposition of su(2n) and control of spin systems, Chem. Phys. 267, 11 (2001).

[11] Y. Chen et al., Qubit Architecture with High Coherence and Fast Tunable Coupling, Phys. Rev. Lett. 113, 220502 (2014).

[12] I. D. Kivlichan, J. McClean, N. Wiebe, C. Gidney, A. Aspuru-Guzik, G. Kin-Lic Chan, and R. Babbush, Quantum Simulation of Electronic Structure with Linear Depth and Connectivity, Phys. Rev. Lett. 120, 110501 (2018). 
[13] E. Farhi and A. W. Harrow, Quantum supremacy through the quantum approximate optimization algorithm, arXiv:1602.07674.

[14] A. Kandala, K. Temme, A. D. Córcoles, A. Mezzacapo, J. M. Chow, and J. M. Gambetta, Error mitigation extends the computational reach of a noisy quantum processor, Nature (London) 567, 491 (2019).

[15] S. Boixo, S. V. Isakov, V. N. Smelyanskiy, R. Babbush, N. Ding, Z. Jiang, M. J. Bremner, J. M. Martinis, and H. Neven, Characterizing quantum supremacy in near-term devices, Nat. Phys. 14, 595 (2018).

[16] F. Arute et al., Quantum supremacy using a programmable superconducting processor, Nature (London) 574, 505 (2019).

[17] C. Neill, A path towards quantum supremacy with superconducting qubits, Ph.D. thesis, University of California Santa Barbara, 2017.

[18] F. Yan, P. Krantz, Y. Sung, M. Kjaergaard, D. L. Campbell, T. P. Orlando, S. Gustavsson, and W. D. Oliver, Tunable Coupling Scheme for Implementing High-Fidelity Two-Qubit Gates, Phys. Rev. Applied 10, 054062 (2018).

[19] M. A. Rol, F. Battistel, F. K. Malinowski, C. C. Bultink, B. M. Tarasinski, R. Vollmer, N. Haider, N. Muthusubramanian, A. Bruno, B. M. Terhal, and L. DiCarlo, Fast, High-Fidelity Conditional-Phase Gate Exploiting Leakage Interference in Weakly Anharmonic Superconducting Qubits, Phys. Rev. Lett. 123, 120502 (2019).

[20] B. Foxen et al., High speed flux sampling for tunable superconducting qubits with an embedded cryogenic transducer, Supercond. Sci. Technol. 32, 015012 (2019).

[21] P. Macha, S. H. W. van der Ploeg, G. Oelsner, E. Ilichev, H.-G. Meyer, S. Wnsch, and M. Siege, Losses in coplanar waveguide resonators at millikelvin temperatures, Appl. Phys. Lett. 96, 062503 (2010).
[22] Y. Shalibo, Y. Rofe, D. Shwa, F. Zeides, M. Neeley, J. M. Martinis, and N. Katz, Lifetime and Coherence of TwoLevel Defects in a Josephson Junction, Phys. Rev. Lett. 105, 177001 (2010).

[23] P. V. Klimov et al., Fluctuations of Energy-Relaxation Times in Superconducting Qubits, Phys. Rev. Lett. 121, 090502 (2018).

[24] See Supplemental Material, which includes Refs. [25-30], at http://link.aps.org/supplemental/10.1103/PhysRevLett .125 .120504 for additional data, analysis, and discussion.

[25] R. Barends et al., Superconducting quantum circuits at the surface code threshold for fault tolerance, Nature (London) 508, 500 (2014).

[26] E. Magesan, J. M. Gambetta, and J. Emerson, Scalable and Robust Randomized Benchmarking of Quantum Processes, Phys. Rev. Lett. 106, 180504 (2011).

[27] A. D. Córcoles, J. M. Gambetta, J. M. Chow, J. A. Smolin, M. Ware, J. Strand, B. L. T. Plourde, and M. Steffen, Process verification of two-qubit quantum gates by randomized benchmarking, Phys. Rev. A 87, 030301 (2013).

[28] Z. Chen et al., Measuring and Suppressing Quantum State Leakage in a Superconducting Qubit, Phys. Rev. Lett. 116, 020501 (2016).

[29] P. J. J. O'Malley et al., Qubit Metrology of Ultralow Phase Noise Using Randomized Benchmarking, Phys. Rev. Applied 3, 044009 (2015).

[30] S. Rahimi-Keshari, M. A. Broome, R. Fickler, A. Fedrizzi, T. C. Ralph, and A. G. White, Direct characterization of linear-optical networks, Opt. Express 21, 13450 (2013).

[31] J. Wallman, C. Granade, R. Harper, and S. T. Flammia, Estimating the coherence of noise, New J. Phys. 17, 113020 (2015).

[32] R. Barends et al., Diabatic Gates for Frequency-Tunable Superconducting Qubits, Phys. Rev. Lett. 123, 210501 (2019). 